\title{
$\$$ Research Square

\section{A metabolic modeling approach reveals promising therapeutic targets and antiviral drugs to combat COVID-19}

\section{Fernando Santos-Beneit}

Institute of Sustainable Processes, Universidad de Valladolid, Spain

Vytautas Raškevičius

Institute of Cardiology, Lithuanian University of Health Sciences, Kaunas, Lithuania

Vytenis A. Skeberdis

Institute of Cardiology, Lithuanian University of Health Sciences, Kaunas, Lithuania

Sergio Bordel ( $\square$ sergio.bordel@uva.es )

Institute of Sustainable Processes, Universidad de Valladolid, Spain

\section{Research Article}

Keywords: covid-19, metabolic modeling, therapeutic intervention, triacsin C, ACSL3, celgosivir

Posted Date: June 23rd, 2020

DOI: https://doi.org/10.21203/rs.3.rs-36346/v1

License: (c) (i) This work is licensed under a Creative Commons Attribution 4.0 International License.

Read Full License

Version of Record: A version of this preprint was published at Scientific Reports on June 7th, 2021. See the published version at https://doi.org/10.1038/s41598-021-91526-3. 


\section{Abstract}

In this study we have developed a metabolic modeling approach to identify human metabolic enzymes which can be targeted for therapeutic intervention against COVID-19. A literature search was carried out in order to identify suitable inhibitors of these enzymes, which were confirmed by docking calculations. In total, 10 targets and 12 bioactive molecules have been predicted. Among the most promising molecules we identified Triacsin C, which inhibits ACSL3, and which has been shown to be very effective against different viruses, including positive-sense single-stranded RNA viruses. Similarly, we also identified the drug Celgosivir, which has been successfully tested in cells infected with different types of viruses such as Dengue, Zika, Hepatitis C and Influenza. Finally, other drugs targeting enzymes of lipid metabolism, carbohydrate metabolism or protein palmitoylation (such as propylthiouracil, 2-bromopalmitate, lipofermata, tunicamycin, benzyl isothiocyanate, tipifarnib and lonafarnib) are also proposed.

\section{Introduction}

The COVID-19 pandemic, caused by the virus SARS-CoV-2, has resulted in a substantial increase in mortality and serious economic and social disruption worldwide. In this context, the rapid identification of drugs against SARS-CoV-2 is essential. Computational methods and systems biology approaches, as the one presented here, can play a significant role in this process of identification of suitable drugs.

In order to inhibit the replication and assembly of viral particles, two main approaches can be attempted: the first one consists in targeting viral proteins involved in replication, the second is based on the fact that many native enzymes of the host are necessary to produce the constitutive biomolecules of viral particles, which makes them potential drug targets. Some of the ways in which viruses reprogram the host metabolism and support viral replication are, among others, activation of glycolysis, fatty acid biosynthesis and elongation, protein glycosylation and inhibition of mitochondrial function (1). It is reasonable to expect that drugs targeting key human metabolic enzymes can be used to inhibit viral replication. Indeed, nucleoside and nucleotide analogs such as Tenovovir, Sofosbuvir or Ribavirin are already being used as antiviral drugs. Pharmacological inhibition of either acetyl-CoA carboxylase (ACC) or fatty acid synthase (FASN) has also been shown to result in a strong reduction of viral titers in vaccinia infection (2).

In many cases, the metabolic resources of the host cell are diverted to the formation of virions via the recruitment of metabolic enzymes and their localization in "replication and transcription complexes" (RTCs), which are vesicles formed from endoplasmic reticulum (ER) membranes, in which the virus replicates. This recruitment takes place via the interaction of host enzymes with viral proteins, such as Nsp3 of rotavirus, which recruits FASN, or Ns5a of hepatitis C virus, which recruits phosphatidylinositol 4kinase alpha (PI4KA) (1). SARS-CoV-2, in the same manner as other coronaviruses, also replicates within ER derived vesicles (3). Therefore, it is reasonable to expect that host enzymes key for viral replication are 
recruited to RTCs via their interaction with viral proteins. A human-SARS-CoV-2 interactome has been recently published (4), which contains 332 high-quality human-SARS-CoV-2 protein-protein interactions, of which 90 involve metabolic enzymes (see supplementary file S1).

The goal of this work is to identify metabolic enzymes that are suitable drug targets against viral replication. In previous articles $(5,6)$ we developed the python library pyTARG, with the object of finding metabolic drug targets against cancer cell lines. This method makes use of a human Genome Scale Metabolic Model (GSMM), which is constrained using RNA-seq data. This allows computing metabolic flux distributions and evaluating the outcomes of restricting enzymatic reaction rates. In order to use this methodology for the evaluation of the metabolic fluxes in lung cells infected by SARS-CoV-2, a literature based stoichiometric equation for the formation of virions (see supplementary material S3) has been added to a previously used human $\operatorname{GSMM}(5,6)$. The metabolic model was constrained based on RNAseq data of lung tissue (obtained from the Human Protein Atlas).

\section{Results And Discussion}

Infection by SARS-CoV-2 has been modeled by making use of the interactions between viral proteins and human enzymes (5). Five of these enzymes (NDUFB9, ETFA, ATP6AP1, ATP6V1A and ATP1B1) are involved in oxidative phosphorylation. These interactions are interpreted as inhibitory or as relocating the involved proteins to RTCs instead of mitochondria, which would suppress their function. This has been modeled by constraining oxidative phosphorylation to have a zero flux in the model of infected cells. For the rest of metabolic enzymes expressed in the lung and interacting with viral proteins, the constraints on their rates have been removed (this is equivalent to assume that their rates could increase as a result of these enzymes being highly concentrated within RTCs). Possible changes in gene expression were modeled by identifying the transcription factors that interact with viral proteins. Only TCF12 was identified. This gene is known to be a transcriptional repressor. Thus, the genes regulated by TCF12 are assumed to be upregulated and the constraints on their reactions were also removed in the model. The model predicts a maximal rate of virion formation of $0.012 \mathrm{nmol}$-virions per hour and gram of dry infected cells. The metabolic model of SARS-CoV-2 infected cells and the model of non-infected lung cells are available at https://github.com/SergioBordel/COVID-19.

The effects of inhibiting the function of each of the enzymes in the virus-host interactome were tested with the function block of pyTARG. This restricts the rate of reactions catalyzed by the tested enzymes to 0.1 times its original value and computes the ratio by which the objective function (the rate of virion production or cellular biomass, for infected and for healthy cells respectively) decreases. This ratio takes values from 1 (indicating no effect) and 0.1 (indicating that the objective function decreases in the same proportion as the flux in the targeted reaction). The model of non-infected lung cells was used as a quantification of potential secondary effects. In order to select suitable targets, a difference between both ratios (non-infected and infected) of 0.3 was used as cutoff value. Using this method, 10 human enzymes were identified (see Table 1). 
A literature search was carried out in order to identify inhibitors of the selected targets, and to evaluate potential secondary effects. When possible, drugs approved or under clinical trials were selected, but we also considered those in a pre-clinical status or under investigation (see references and structures of these compounds in supplementary files S2-S3). The literature survey yielded a total of 10 compounds (see Table 1). The affinity of the identified drugs for some the target enzymes (those for which a reliable 3D structure could be obtained) was further validated using docking. Docking was also used for the identification of putative inhibitors for enzymes without known inhibitors, as it is the case of Tipifarnib and Lonafarnib, predicted to bind FAR2 (Figure 1).

Table 1. Table with the selected target enzymes for SARS-CoV-2 treatment. See an extended version of Table 1 in Supplementary file S2. **These compounds have been identified in our docking analyses rather than in the literature revision approach.

$\begin{array}{lllll}\begin{array}{l}\text { Viral-host } \\ \text { interaction }\end{array} & \begin{array}{l}\text { Ensembl ID } \\ \text { (human gene) }\end{array} & \text { Definition } & \begin{array}{l}\text { Cellular } \\ \text { Process }\end{array} & \text { Known Inhibitors } \\ & & & \end{array}$

Orf3a-

ALG5

ENSG00000120697

dolichyl-phosphate

beta-

glucosyltransferase

$\begin{array}{ll}\text { Orf9c- } & \text { ENSG00000159063 } \\ \text { ALG8 } & \end{array}$

$\begin{array}{ll}\text { Nsp4- } & \text { ENSG00000253710 } \\ \text { ALG11 } & \end{array}$

$\begin{array}{lll}\begin{array}{l}\text { Nsp7- } \\ \text { CYB5R3 }\end{array} & \text { ENSG00000100243 } & \begin{array}{l}\text { NADH cytochrome-b5 } \\ \text { reductase }\end{array}\end{array}$

\section{Spike- ZDHHC5}

ENSG00000156599

\section{Nsp2-}

SLC27A2

$\begin{array}{ll}\text { Nsp7- } & \text { ENSG00000123983 } \\ \text { ACSL3 } & \end{array}$

$\begin{array}{ll}\text { Nsp7- } & \text { ENSG00000018510 } \\ \text { AGPS } & \end{array}$

alpha-1,3-
glucosyltransferase

$$
\text { alpha-1,2- }
$$

mannosyltransferase

$\mathrm{N}-$-Glycan
biosynthesis

Celgosivir;

Tunicamycin

$\begin{array}{ll}\mathrm{N} \text {-Glycan } & \text { Celgosivir; } \\ \text { biosynthesis } & \text { Tunicamycin }\end{array}$

$\begin{array}{ll}\begin{array}{l}\text { N-Glycan } \\ \text { biosynthesis }\end{array} & \text { Celgosivir; } \\ \text { Tunicamycin }\end{array}$

$\begin{array}{ll}\text { Sugar } & \text { Propylthiouracil; } \\ \text { metabolism } & \text { ZINC-39395747; } \\ & \text { ZINC-05626394 }\end{array}$
palmitoyltransferase 5
Protein palmitoylation
2-bromopalmitate

Lipid Lipofermata
metabolism

$\begin{array}{ll}\text { solute carrier family } & \text { Lipid } \\ 27 \text { member } 2 & \text { metabolism }\end{array}$

long-chain acyl-CoA synthetase

Lipid
metabolism

$\begin{array}{ll}\text { alkylglycerone } & \text { Lipid } \\ \text { phosphate synthase } & \text { metabolism }\end{array}$

Lipid

metabolism

Triacsin C

Benzyl isothiocyanate;

ZINC-69435460

\begin{tabular}{lllll}
$\begin{array}{l}\text { Orf9c- } \\
\text { FAR2 }\end{array}$ & ENSG00000064763 & $\begin{array}{l}\text { alcohol-forming fatty } \\
\text { acyl-CoA reductase }\end{array}$ & $\begin{array}{l}\text { Lipid } \\
\text { metabolism }\end{array}$ & $\begin{array}{l}\text { Tipifarnib*; } \\
\text { Lonafarnib* }\end{array}$ \\
$\begin{array}{l}\text { Nsp7- } \\
\text { NAT14 }\end{array}$ & ENSG00000090971 & $\begin{array}{l}\text { putative N- } \\
\text { acetyltransferase 14 }\end{array}$ & $\begin{array}{l}\text { Not } \\
\text { determined }\end{array}$ & Not found \\
\hline
\end{tabular}


Some of the 10 putative targets are known oncogenes for which drugs are already in clinical trials (AGPS, SLC27A2 and ACSL3). AGPS is inhibited by benzyl isothiocyanate (BITC), the anti-fungal agent Antimycin $A$ and the investigational new drug ZINC-69435460 [3-(2-fluorophenyl)-N-(1-(2-oxo2,3-dihydro-1Hbenzo[d]imidazol-5-yl)ethyl)butanamide]. Antimycin A can be discarded due to its cytotoxicity, as it is also an inhibitor of cytochrome $C$ reductase, essential for eukaryotic cells (7). The two other inhibitors could be promising alternatives. BITC inhibits the proliferation of human glioma U87MG and hepatic carcinoma HepG2 cells, as well as aggressive human breast tumors, and ZINC-69435460, makes multiple specific interactions with residues in the AGPS active site, and inhibits its activity (8). SLC27A2 (also known as FATP2) is a transmembrane transporter coenzyme that participates in the long-chain fatty acid betaoxidation and peroxidase lipid metabolism. In a recent publication it has been shown that the selective pharmacological inhibition of FATP2 with lipofermata (5-bromo-5'-phenyl-spiro[3H-indole-3,2' $\left(3^{\prime} H\right)$ $[1,3,4]$ thiadiazol]-2(1H)-one) suppresses the activity of pathologically activated neutrophils and substantially reduces melanoma growth and invasion (9). ACSL3 is one of the five members of the long chain acyl-CoA synthetase (ACSL) family. Specifically, ACSL3 has been identified as a host factor required for Poliovirus replication, which, as SARS-CoV-2, is a positive-sense single-stranded RNA virus that replicates its genome in association with membranes (10). Inhibition of ACSL3 with inhibitors such as Triacsin $\mathrm{C}$ has been shown to reduce virion formation in human cells infected with different viruses, including cytomegalovirus, rotavirus and hepatitis $C(11,12)$. For these reasons, and according to our metabolic modelling results, this drug constitutes a very promising therapeutic option to treat SARS-CoV2 infection.

ZDHHC5 has been found to interact with the Spike protein of SARS-CoV-2 (5). The protein ZDHHC5 is involved in the palmitoylation of proteins, which plays an important role in protein-membrane interactions, protein trafficking and enzyme activity by contributing to their membrane association. Palmitoylation of the SARS-CoV-Spike protein has been reported to facilitate its fusion with the host cellular membrane, which clearly suggests ZDHHC5 as potential target for therapeutic inhibition of SARS$\mathrm{CoV}$ and other coronaviruses such as SARS-CoV-2. In vitro experiments have revealed some molecules that inhibit cellular processes associated with palmitoylation, with one of the best examples being 2Bromopalmitate (13).

The enzyme NADH cytochrome-b5 reductase (i.e. CYB5R3) is inhibited by the approved drug, propylthiouracil (PTU) (14). PTU is an antithyroid medication used in the therapy of hyperthyroidism and the Graves disease. It is still unclear how PTU exerts its inhibitory effects on CYB5R3 at a molecular level. Nevertheless, a repurposing strategy for COVID-19 treatment may merit investigation. Other described inhibitors of CYB5R3 are the investigational new drugs ZINC-39395747 and ZINC-05626394, which have been shown to be more potent inhibitors of CYB5R3 than PTU (15); matching with our docking results (see Figure 2).

The rest of the identified targets are involved in N-glycan biosynthesis and glycosylation. Glycosylation is vital in the maturation process of viral proteins. The Spike protein of SARS-CoV-2 has 16 glycosylation residues (16). The importance of protein glycosylation is evidenced by the use of Celgosivir (an inhibitor 
of $\mathrm{N}$-glycan synthesis) in treating cells that harbour Dengue virus, which prevents proper protein glycosylation, leading to protein misfolding and impaired replicative efficiency (17). Celgosivir (and also other iminosugars such as deoxynojirimycin or castanospermine) has been shown to be a very promising drug, not only for the treatment of Dengue virus or members of the same family (like for example ZIKV), but also for other viruses belonging to different families, such as the hepatitis $\mathrm{C}$ or Influenza viruses (1820). As 3 of our 10 selected enzyme targets are involved in the N-Glycan biosynthesis pathway (i.e. ALG5, ALG8, ALG11) we propose treating SARS-CoV-2 with inhibitors of glycoprotein biosynthesis such as the previous mentioned iminosugars, or the nucleoside antibiotic tunicamycin, which also inhibits N-linked protein glycosylation. Interestingly, this compound has been shown to display strong antiviral activity against certain viruses like for example the Epstein-Barr virus (21). Similarly, drugs that disrupt the integrity of the Golgi apparatus (monensin, brefeldin A, bafilomycin, nocodazole) also alters glycoprotein synthesis and could be also effective for treating COVID-19.

\section{Materials And Methods}

\section{Stoichiometric equation of SARS-CoV-2 virions:}

The SARS-CoV-2 genome is very similar to SARS-CoV, each has an Orf1ab encoding 16 predicted nonstructural proteins (Nsp1-16) and each has the 4 typical coronavirus structural proteins, including: Spike (S), Envelope (E), Membrane (M) and Nucleocapsid (N). Thus, the structural information from SARS-CoV, obtained by crio-electron microscopy (16), has been used to estimate the stoichiometric relations between the main structural proteins. Image analysis revealed a relation of $8 \mathrm{M}$ protein dimers per $\mathrm{S}$ protein trimer in the virion surface, with an estimated number of 90 spikes per viral particle. $\mathrm{N}$ proteins are found both at the surface and within the virion, with $\mathrm{M}: \mathrm{N}$ ratios between 1:1 and 3:1 depending on the authors (22). The M:S ratio determined by crio-electron microscopy is equal to 5.3 and corresponds to the inverse of the ratio between the two protein lengths, which could be consistent with viral proteins being translated at the same rate per amino acid. This would lead to a M:N ratio of 1.9 , which is within the previously mentioned rank and has been chosen for modeling purposes. No other proteins have been included in the structure of the virion, E protein seems to play a key role in virion assembly, but it is not present in significant quantities in viral particles. The S protein of SARS-CoV-2 has $16 \mathrm{~N}$-glycosylation residues (23) and one palmitoyl residue, while the $\mathrm{M}$ protein has one $\mathrm{N}$-glycosylation residue and one palmitoyl residue. The lipid quantity of phospholipids per virion was estimated by considering an average virion diameter of 125 $\mathrm{nm}$ and assuming that the surface proteins are packed as circles touching each other forming a hexagonal pattern (22). This geometry leaves $10 \%$ of the surface for a lipid bilayer, whose composition has been assumed to be equal to that of the human endoplasmic reticulum (24). In a previous work (25) the authors evaluated the conductance of purified protein SARS-CoV-3a in planar lipid bilayers with a biologically relevant mix with a composition to that of intracellular organelle membranes, such as the endoplasmic reticulum (ER)-Golgi intermediate compartment (ERGIC). Thus, this assumption appears to be widely accepted. 
Not only the structural proteins forming the virions are being translated, but all the viral proteins, however only the structural proteins are being drained away from the cells when virions are secreted, the rest of the proteins are assumed to be degraded within the cell at the same rate as they are produced, and their aminoacids are constantly recycled, this does not result in a drain of amino acids from the cells, but it has energetic costs in form of ATP and GTP. In order to have an approximate estimation of these costs we assume that all the viral proteins are translated at a rate inversely proportional to its length. Equally. Genomic RNA and sub-genomic mRNAs require methylation, which consumes SAM units. This has been modeled by assuming that the genomic and sub-genomic mRNAs are all produced in the same proportions. This choice does not have any impact on the conclusions of the article.

The detailed virion composition can be found in the supplementary material S3.

\section{Metabolic modeling:}

A human genome scale metabolic model has been constrained using the fullconstrain function from pyTARG (https://github.com/SergioBordel/pyTARG), as described in reference (6). The lung RNA-seq data necessary to constrain the model were obtained from the Human Protein Atlas (www.proteinatlas.org). In order to model infection by SARS-CoV-2, oxidative phosphorylation has been constrained to have a zero rate. Attenuation of respiration is known to be a general feature of viral infection (1). In our case this is further supported by the fact that 5 genes involved in oxidative phosphorylation (NDUFB9, ETFA, ATP6AP1, ATP6V1A and ATP1B1) are part of the human-SARS-CoV-2 interactome (4). These interactions are interpreted as inhibitory or as relocating the involved proteins to RTCs instead of mitochondria, which would suppress their function. For the rest of metabolic enzymes expressed in lung and interacting with viral proteins, the constraints on their rates have been removed (this is equivalent to assume that their rates could increase as a result of these enzymes being highly concentrated within RTCs). Possible changes in gene expression were modeled by identifying the transcription factors that interact with viral proteins. Only TCF12 was identified. This gene is known to be a transcriptional repressor. Thus, the genes regulated by it are assumed to be upregulated and the constraints on the reactions that they catalyze were removed in the model. The list of human transcription factors and the genes regulated by them were obtained from the TRANSFAC database. The metabolic model of SARS-CoV-2 infected cells and the model of non-infected lung cells are available at https://github.com/SergioBordel/COVID-19. Both models are in SBML format and can be used to compute distributions of metabolic fluxes using the function flux from pyTARG.

\section{Identification of drug targets:}

The effects of blocking metabolic enzymes were modeled by selecting virion production as objective function and using the function block from pyTARG. This function reduces the flux in the selected metabolic reaction to 0.1 times its original value and computes the ratio by which virion production is reduced as a result of this restriction in the metabolic flux. In order to evaluate the effects on non-infected cells, the lung metabolic model was used and production of cell biomass was chosen as an objective instead. 
Calculation of protein structures:

Tridimensional structures of the target proteins were generated using the SWISS-MODEL cloud based modeling tool (26) in the cases of: 000116 (template PDB ID 4BC7), 095573 (template PDB ID 5MSS), Q2TAA5 (template PDB ID Q2TAA5), Q8WUY8 (template PDB ID 5L7J), Q9Y673 (template PDB ID 5MLZ), 014975 (template PDB ID 3A9V), and Q9C0B5 (template PDB ID 6BMS). Q9BVK2 was modelled with ITASSER (27). Q96K12 was modelled with Robetta (28). QMEAN was used to assess homology model quality (29).

\section{Docking:}

Q8IVS2 (PDB ID 2C2N) and P00387 (PDB ID 1UMK) structures were acquired directly from RCSB PDB. As other human structures were not available, homology models were used instead. Protein model structures were prepared initially with UCSF Chimera (30) and then converted to pdbqt file format with Open Babel (31). Cofactor biding sites on PDB structures and templates were used as potential docking locations to set up cubic docking calculations. The calculations were performed in boxes of $20 \AA^{3}$. The conformer models of the ligands were acquired from PubChem (32) or ZINC (33) databases and then converted to pdbqt file format with Open Babel (31). QuickVina-W was used to carry out docking task with all prepared structural data (34). Docking results were visualized, analyzed and final docking images were prepared with UCSF Chimera (30).

\section{Declarations}

\section{Acknowledgments}

This work has been supported by a grant from the Lithuanian Research Council (S-SEN-20-6). The financial support from the Regional Government of Castilla y León and the FEDER program is also gratefully acknowledged (CLU 2017-09 and VA281P18). Fernando Santos-Beneit contract is supported by NESTLE throughout FUNGE.

\section{References}

1. M. Goodwin, S. Xu, J. Munger. Stealing the keys of the kitchen: viral manipulation of the host cell metabolic network. Trends Microbiol. 23, 789-798 (2015)

2. D. Greseth, P. Traktman. De novo fatty acid biosynthesis contributes significantly to establishment of a bioenergetically favorable environment for vaccinia virus infection. PLoS Pathog. 10, e1004021 (2014)

3. C. Hagemeijer, I. Monastyrska, J. Griffith, P. van der Sluijs, J. Voortman, et. al. Membrane rearrangements mediated by coronavirus nonstructural proteins 3 and 4 . Virology. 458-459, 125-135 (2014) 
4. E. Gordon, G. M. Jang, M. Bouhaddou, J. Xu, K. Obernier, et. al. A SARS-CoV-2-Human Protein-Protein Interaction Map Reveals Drug Targets and Potential Drug-Repurposing. Nature. (2020). https://doi.org/10.1038/s41586-020-2286-9

5. Raškevičius, V. Mikalayeva, I. Antanavičiūtè, I. Ceslevičienė, V.A. Skeberdis, et. al. Genome scale metabolic models as tools for drug design and personalized medicine. PlosOne. 13, e0190636 (2018)

6. Bordel. Constraint based modeling of metabolism allows finding metabolic cancer hallmarks and identifying personalized therapeutic windows. Oncotarget. 9, 19716-19729 (2018)

7. S. Huang, D. Cobessi, E. Y. Tung, E. A. Berry, Binding of the respiratory chain inhibitor antimycin to the mitochondrial bc1 complex: a new crystal structure reveals an altered intramolecular hydrogenbonding pattern. J Mol Biol. 351, 573-597 (2005).

8. Piano, D. I. Benjamin, S. Valente, S. Nenci, B. Marrocco, et. al. Discovery of Inhibitors for the Ether Lipid-Generating Enzyme AGPS as Anti-Cancer Agents. ACS Chem Biol. 10, 2589-2597 (2015).

9. Veglia, V. A. Tyurin, M. Blasi, A. De Leo, A. V. Kossenkov, et. al. Fatty acid transport protein 2 reprograms neutrophils in cancer. Nature. 569, 73-78 (2019).

10. A. Nchoutmboube, E. G. Viktorova, A. J. Scott, L. A. Ford, Z. Pei, et. al. Increased long chain acyl-Coa synthetase activity and fatty acid import is linked to membrane synthesis for development of picornavirus replication organelles. PLoS Pathog. 9, e1003401 (2013).

11. M. Liefhebber, C. V. Hague, Q. Zhang, M. J. Wakelam, J. McLauchlan, Modulation of triglyceride and cholesterol ester synthesis impairs assembly of infectious hepatitis C virus. J Biol Chem. 289, 2127621288 (2014).

12. Kim, D. George, A. M Prior, K. Prasain, S. Hao, et. al. Novel Triacsin C Analogs as Potential Antivirals against Rotavirus Infections. Eur J Med Chem. 50, 311-318 (2012).

13. C. Jennings, M. J. Nadolski, Y. Ling, M. B. Baker, M. L. Harrison, et. al. 2-Bromopalmitate and 2-(2hydroxy-5-nitro-benzylidene)-benzo[b]thiophen-3-one inhibit DHHC-mediated palmitoylation in vitro. J Lipid Res. 50, 233-242 (2009).

14. Lee, K. Kariya, Propylthiouracil, a Selective Inhibitor of NADH-cytochrome b5 Reductase. FEBS Lett. 209, 49-51 (1986).

15. Rahaman, F. G. Reinders, D. Koes, A. T. Nguyen, S. M. Mutchler, et al. Structure Guided Chemical Modifications of Propylthiouracil Reveal Novel Small Molecule Inhibitors of Cytochrome b5 Reductase 3 That Increase Nitric Oxide Bioavailability. J Biol Chem. 290, 16861-16872 (2015).

16. C. Walls, Y. J. Park, M. A. Tortorici, A. Wall, A. T. McGuire, et. al. Structure, Function, and Antigenicity of the SARSCoV-2 Spike Glycoprotein. Cell. 180, 1-12 (2020).

17. P. S. Rathore, P. N. Paradkar, S. Watanabe, K. H. Tan, C. Sung, et al. Celgosivir Treatment Misfolds Dengue Virus NS1 Protein, Induces Cellular Pro-Survival Genes and Protects Against Lethal Challenge Mouse Model. Antiviral Res. 92, 453-460 (2011).

18. Sung, Y. Wei, S. Watanabe, H. S. Lee, Y. M. Khoo, et al. Extended Evaluation of Virological, Immunological and Pharmacokinetic Endpoints of CELADEN: A Randomized, Placebo-Controlled 
Trial of Celgosivir in Dengue Fever Patients. PLoS Negl Trop Dis. 10, e0004851 (2016).

19. Bhushan, L. Lim, I. Bird, S. K. Chothe, R. H. Nissly, S. V. Kuchipudi, Iminosugars With Endoplasmic Reticulum a-Glucosidase Inhibitor Activity Inhibit ZIKV Replication and Reverse Cytopathogenicity in vitro. Front Microbiol. 11, 531 (2020).

20. Durantel. Celgosivir, an Alpha-Glucosidase I Inhibitor for the Potential Treatment of HCV Infection. Curr Opin Investig Drugs. 10, 860-870 (2009).

21. M. Hutt-Fletcher, N. Balachandran, P. A. LeBlanc, Modification of Epstein-Barr virus replication by tunicamycin. J Virol. 57, 117-123 (1986).

22. W. Neuman, G. Kiss, A. H. Kunding, D. Bhella, M. Fazil Baksh, et. al. A structural analysis of M protein in coronavirus assembly and morphology. J. Struct. Biol. 174, 11-22 (2011).

23. C. Walls, Y. J. Park, M. A. Tortorici, A. Wall, A. T. McGuire, et. al. Structure, Function, and Antigenicity of the SARSCoV-2 Spike Glycoprotein. Cell. 180, 1-12 (2020).

24. Casares, P. V. Escriba, C. A. Rossello. Membrane Lipid Composition: Effect on Membrane and Organelle Structure, Function and Compartmentalization and Therapeutic Avenues. Int. J. Mol. Sci. 20, 2167 (2019).

25. Castaño-Rodriguez, J.M. Honrubia, J. Gutiérrez-Álvarez, M. L. DeDiego, J. L. Nieto-Torres, et. al. Role of Severe Acute Respiratory Syndrome Coronavirus Viroporins E, 3a, and 8a in Replication and Pathogenesis. mBio, e02325-17 (2018).

26. Waterhouse, M. Bertoni, S. Bienert, G. Studert, G. Tauriello et al., SWISS-MODEL: homology modelling of protein structures and complexes. Nucleic Acids Res, 46, W296-W303 (2018).

27. Yang, Y. Zhang, I-TASSER server: new development for protein structure and function predictions. Nucleic Acids Res. 43, W174-81 (2015).

28. Song, F. DiMaio, R. Y. Wang, D. Kim, C. Miles et al., High-resolution comparative modeling with RosettaCM. Structure. 21, 1735-1742 (2013).

29. Benkert, M. Biasini, T. Schwede, Toward the estimation of the absolute quality of individual protein structure models. Bioinformatics. 27, 343-350 (2010).

30. F. Pettersen, T. D. Goddard, C. C. Huang, G. S. Couch, D. M. Greenblat. et al., UCSF Chimera-a visualization system for exploratory research and analysis. J Comput Chem. 25, 1605-1612 (2004).

31. M. O'Boyle, M. Banck, C. A. James, C. Morley, T. Vandermeersch et al., Open Babel: An open chemical toolbox. Journal of Cheminformatics. 3, 33 (2011).

32. Kim. Getting the most out of PubChem for virtual screening. Expert Opin Drug Discov. 11, $843-855$ (2016).

33. Sterling, J.J. Irwin. ZINC 15 - Ligand Discovery for Everyone. Journal of Chemical Information and Modeling. 55, 2324-2337 (2015).

34. M. Hassan, A. A. Alhossary, Y. Mu, C. K. Mwoh. Protein-Ligand Blind Docking using QuickVina-W with Inter-Process Spatio-Temporal Integration. Scientific Reports. 7, 15451 (2017). 


\section{Figures}

A
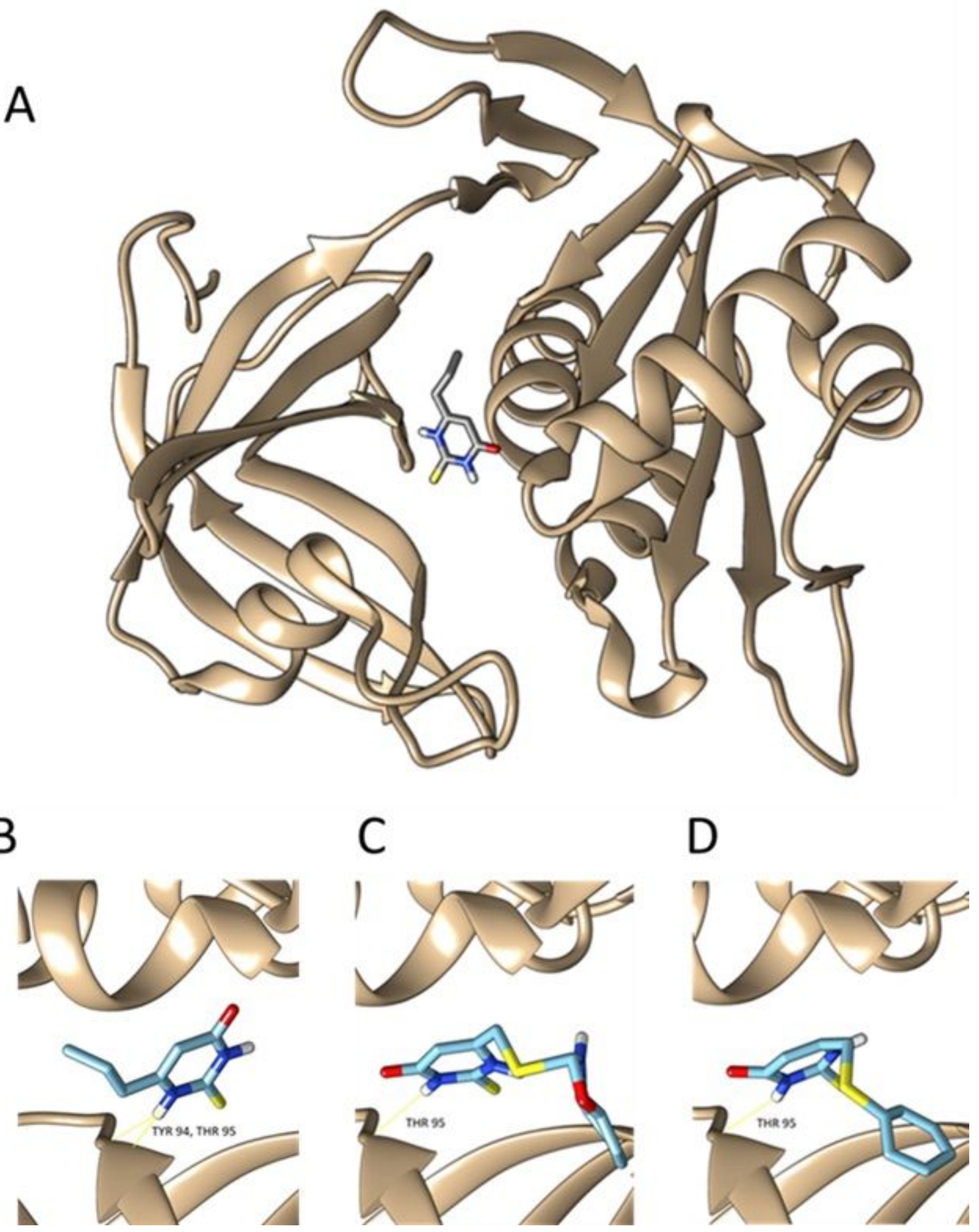

Figure 1

Structure of CYB5R3 (A), binding site of Propylthiouracil (PTU), with a binding $\Delta \mathrm{G}$ of $-5.4 \mathrm{kcal} / \mathrm{mol}$ (B) and docking of ZINC39395747 ( $\Delta \mathrm{G}=-7.5 \mathrm{kcal} / \mathrm{mol})(\mathrm{C})$ and ZINC05626394 ( $\Delta \mathrm{G}=-6.5 \mathrm{kcal} / \mathrm{mol})(\mathrm{D})$. 
A

a Healthy lung cells $\quad$ Infected lung cells

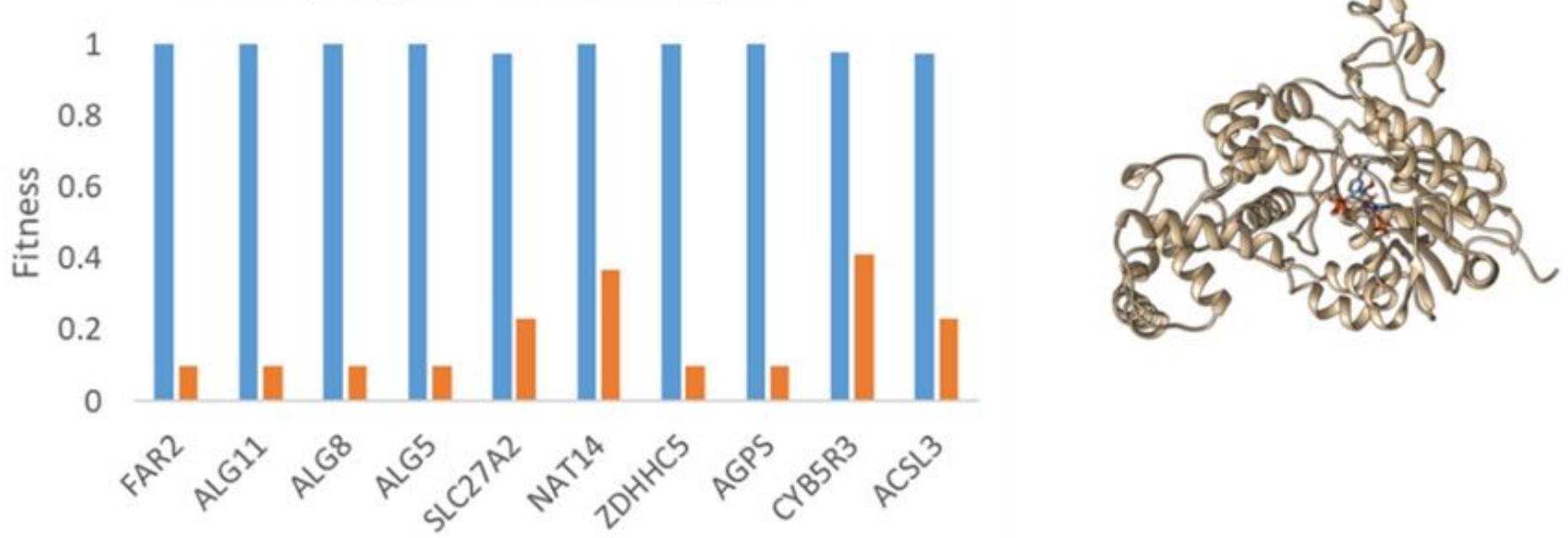

C

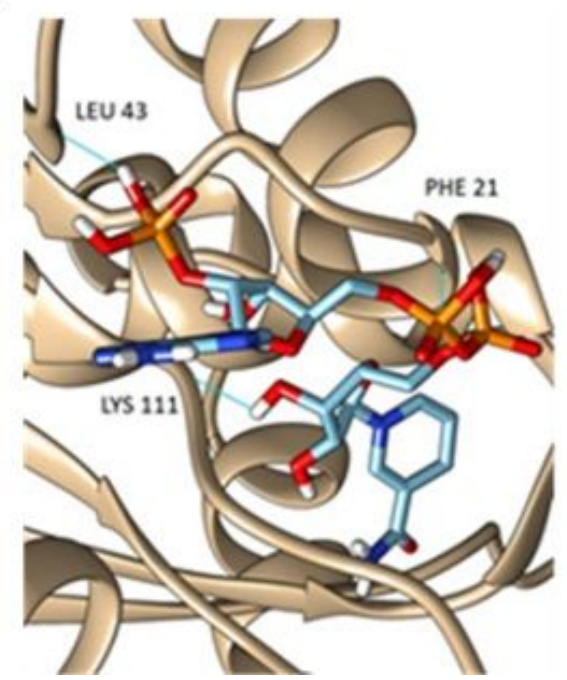

D

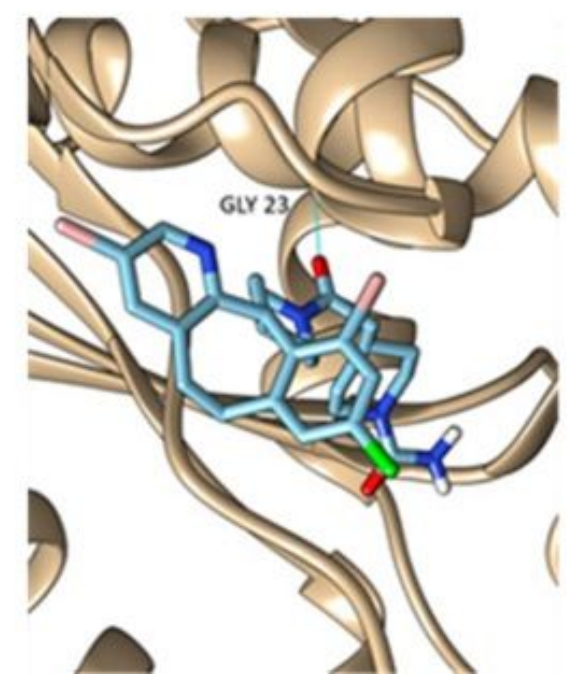

B

$\mathrm{E}$

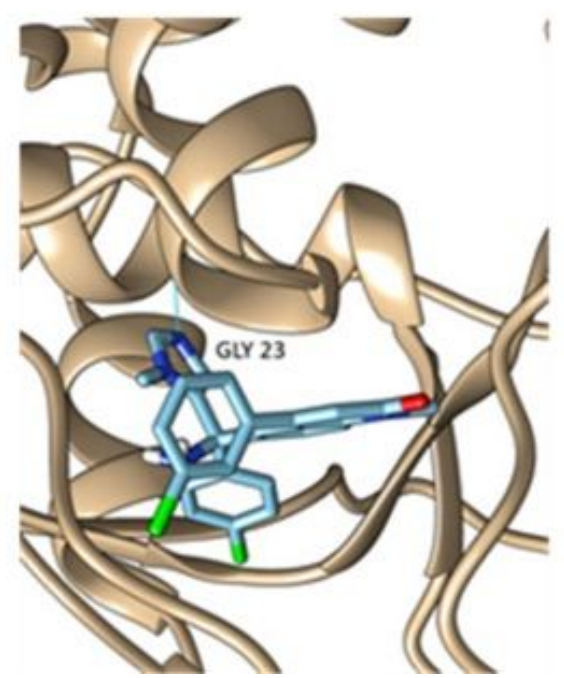

Figure 2

Predicted effects of blocking metabolic enzymes, on the healthy lung cells and the infected cells respectively (A). Fatty acyl-CoA reductase (FAR2) bound to NADPH (B). Docking revealed a binding affinity of $-9.5 \mathrm{kcal} / \mathrm{mol}$ for FAR2 and NADPH, one of its natural substrates (C), while the predicted binding affinities of Lonafarnib (D) and Tipifarnib (E), are $-10.9 \mathrm{kcal} / \mathrm{mol}$ and $-9.5 \mathrm{kcal} / \mathrm{mol}$ respectively. This suggests that these two drugs (with a preference for Lonafarnib) could displace NADPH from its binding site and block the activity of FAR2.

\section{Supplementary Files}

This is a list of supplementary files associated with this preprint. Click to download.

- SupplementaryDataS3.docx 